\title{
miR-181a-5p regulates the proliferation and apoptosis of glomerular mesangial cells by targeting KLF6
}

\author{
$\mathrm{XINYUE} \mathrm{LIANG}^{1}$ and WEN $\mathrm{XU}^{2}$ \\ ${ }^{1}$ Department of Geriatrics, Renji Hospital, School of Medicine, Shanghai Jiao Tong University; \\ ${ }^{2}$ State Key Laboratory of Bioreactor Engineering and Shanghai Key Laboratory of New Drug Design, School of Pharmacy, \\ East China University of Science and Technology, Shanghai 200127, P.R. China
}

Received April 24, 2019; Accepted December 12, 2019

DOI: $10.3892 /$ etm. 2020.8780

\begin{abstract}
Diabetic nephropathy (DN) is a chronic loss of kidney function that frequently occurs in patients with diabetes mellitus and is characterized by abnormal glomerular mesangial cell (GMC) proliferation and apoptosis. By using microarray analysis, microRNA (miR)-181a-5p has previously been identified to be dysregulated in DN. The present study aimed to determine the underlying molecular mechanisms and function of miR-181a-5p in GMCs under DN conditions. First, reverse transcription-quantitative PCR was performed to detect miR-181a-5p and kruppel-like factor 6 (KLF6) expression in GMCs following high-glucose treatment. Subsequently, MTT and flow cytometric assays were performed in order to determine the effect of miR-181a-5p and KLF6 on high-glucose-driven GMC proliferation and apoptosis. After confirming that KLF6 was a target gene of miR-181a-5p via a bioinformatics analysis and luciferase reporter assay, the mRNA and protein expression levels of associated factors in different treatment groups were measured. The results demonstrated that miR-181a-5p was significantly downregulated, while KLF6 was significantly upregulated in GMCs following treatment with high glucose. Furthermore, overexpression of miR-181a led to suppression of cell proliferation and promoted apoptosis of GMCs induced by high glucose, while these effects were inhibited by co-transfection with KLF6. Finally, miR-181-5p was demonstrated to inhibit the expression of KLF6, Bcl-2, Wnt1 and $\beta$-catenin, while increasing the expression levels of Bax and caspase-3. In conclusion, the expression levels of miR-181a-5p were downregulated in GMCs following treatment with high glucose and overexpression of miR-181a-5p may inhibit GMC proliferation and promote apoptosis, at least partially through targeting KLF6
\end{abstract}

Correspondence to: Dr Xinyue Liang, Department of Geriatrics, Renji Hospital, School of Medicine, Shanghai Jiao Tong University, 160 Pujian Road, Shanghai 200127, P.R. China

E-mail: xinyue209@yandex.com

Key words: microRNA-181a-5p, proliferation, apoptosis, glomerular mesangial cells, diabetic nephropathy, kruppel-like factor 6 via the Wnt/ $\beta$-catenin signaling pathway. Overall, the results of the present study suggest that miR-181a-5p may have a crucial role in the occurrence and development of DN and may be a valuable diagnostic marker and therapeutic target for DN.

\section{Introduction}

MicroRNAs (miRNAs/miRs) are small non-coding RNAs that may endogenously regulate gene expression by partially binding to the 3'-untranslated region (3'UTR) of their target mRNAs $(1,2)$. Previous studies have reported that miRNAs are involved in cell differentiation, development and carcinogenesis $(3,4)$.

Diabetic nephropathy (DN) is the chronic loss of kidney function that frequently occurs in patients with diabetes mellitus $(5,6)$. Although the accurate molecular mechanisms underlying the occurrence and progression of DN have remained to be fully elucidated, previous studies have reported that increased mesangial cell proliferation and abnormal apoptosis are major pathological features of early-stage DN $(7,8)$. Recently, a number of studies have demonstrated that certain miRNAs exhibit aberrant expression in the kidneys of mice with DN (9-11). Furthermore, miR-181-5p has been reported to be dysregulated in diabetes mellitus $(12,13)$. Kruppel-like factors (KLFs), a family of DNA-binding transcriptional regulators, are involved in cellular processes (14). KLF6 may function as a putative tumor suppressor in various cancer types, including prostate (15) and colorectal cancer (16). A study indicated that fibrotic kidneys have increased KLF6 expression (17). However, the interaction between miR-181a-5p and KLF6, the roles of either factor on its own in $\mathrm{DN}$, or their interaction in $\mathrm{DN}$-associated processes have remained elusive.

The aim of the present study was to identify the underlying function and molecular mechanisms of miR-181a-5p in DN. In particular, the anti-proliferative and pro-apoptotic effects of miR-181a-5p on high glucose treated glomerular mesangial cells (GMCs) were assessed.

\section{Materials and methods}

Cell culture and treatment. The GMC line SV40-MES-13 (no. YS-ATCC311) was purchased from Shanghai Yansheng 
Industrial Co., Ltd. The cells were cultured in Dulbecco's modified Eagle's medium (DMEM; containing 5.6 nM glucose; Invitrogen; Thermo Fisher Scientific, Inc.) supplemented with 10\% fetal bovine serum (10099-1741; Gibco; Thermo Fisher Scientific, Inc.) in a humidified atmosphere containing $5 \%$ $\mathrm{CO}_{2}$ at $37^{\circ} \mathrm{C}$. A total of $100 \mathrm{U} / \mathrm{ml}$ penicillin and $100 \mu \mathrm{g} / \mathrm{ml}$ streptomycin (Thermo Fisher Scientific, Inc.) were added to the medium in order to prevent bacterial contamination. After $24 \mathrm{~h}$ of pre-incubation in the presence of normal glucose levels (DMEM containing $5.6 \mathrm{nM}$ glucose), further glucose (25 nM glucose; Sigma Aldrich; Merck KGaA) was added to provide a high-glucose (total $31.6 \mathrm{nM}$ glucose) environment (18), and the cells were incubated for another $24 \mathrm{~h}$ at $37^{\circ} \mathrm{C}$ in a humidified atmosphere with $5 \% \mathrm{CO}_{2}$. Following high-glucose treatment, the expression levels of miR-181a-5p and KLF6 were measured accordingly.

Cell transfection. Following treatment, the cells were transfected with miR-181a-5p mimics and control mimics (Shanghai Gene Pharma Co., Ltd.) using Lipofectamine ${ }^{\circledR}$ 2000 (Invitrogen; Thermo Fisher Scientific, Inc.). In order to determine the transfection efficiency of miR-181a-5p, cells were divided into three groups: i) Control group, untransfected cells; ii) negative control (NC) group, cells were transfected with miR-181a-5p mimics NC; and iii) mimics group, cells were transfected with miR-181a-5p mimics. The sequences were as follows: miR-181a-5p mimics, 5'-AACAUUCAACGC UGUCGGUGAGU-3' and control mimics, 5'-UUUGUACUA CACAAAAGUACUG-3'.

Plasmid construction. A KLF6-expressing vector was constructed by inserting the KLF6 gene into the pcDNA3.1 vector. In brief, the sequence for KLF6 was purchased from GenePharma (Shanghai GenePharma Co., Ltd.) and inserted into the pcDNA3.1 vector (GeneChem). In order to determine the effects of KLF6 transfection, cells were divided into three groups: i) Control group, untransfected cells; ii) pcDNA3.1 group, cells transfected with pcDNA3.1; and iii) KLF6 group, cells transfected with pcDNA3.1-KLF6. The human KLF6 sequence is forward, 5'-CTCTCAGCCTGGAAGCTTTTA GCCTAC-3' and reverse, 5'-ACAGCTCCGAGGAACTTT CTCCCA-3'.

MTT assay. In order to determine the effect of the vectors on the amount of viable cells, the high-glucose-treated cells were divided into four groups: i) ontrol group, untransfected cells; ii) NC group, cells were transfected with miR-181a-5p mimics NC; iii) mimics group, cells were transfected with miR-181a-5p mimics; and iv) mimics + KLF6 group, cells were transfected with miR-181a-5p mimics and pcDNA3.1-KLF6. An MTT assay (Sigma-Aldrich; Merck KGaA) was applied to determine the cell viability. In brief, the treated cells were seeded in a 96-well plate at a density of $1 \times 10^{4}$ cells/well. Subsequently, $20 \mu 1$ MTT was added to each well at a concentration of $5 \mathrm{mg} / \mathrm{ml}$ after 12,24 or $48 \mathrm{~h}$ of culture, followed by further incubation for $4 \mathrm{~h}$ at $37^{\circ} \mathrm{C}$ in a humidified atmosphere with $5 \% \mathrm{CO}_{2}$. Following the removal of all medium, dimethylsulfoxide $(100 \mu \mathrm{l})$ was added to each well, followed by shaking for 5 min until the formazan crystals had dissolved completely. The absorbance was measured at $490 \mathrm{nM}$ using a microplate reader (M680-UV Spectrophotometer; Bio-Rad Laboratories, Inc.).

Flow cytometry. An Annexin V-FITC/PI Apoptosis Detection kit (Keygen Biotech) was used to assess the apoptosis rate. In brief, the treated cells were seeded into a 6 -well plate at a density of $4 \times 10^{5}$ cells/well and re-suspended in $500 \mu \mathrm{l}$ binding buffer after $24 \mathrm{~h}$ incubation. Subsequently, $5 \mu \mathrm{l}$ Annexin $\mathrm{V}$-FITC and $5 \mu \mathrm{l}$ propidium iodide were added to the suspension, followed by incubation for $20 \mathrm{~min}$ at room temperature in a humidified atmosphere with $5 \% \mathrm{CO}_{2}$ in the dark. Finally, cells were analyzed using flow cytometry (Beckman Coulter, Inc.) according to the manufacturer's protocol.

Reverse transcription-quantitative PCR (RT-qPCR). Total RNA was isolated from cells using TRIzol ${ }^{\circledR}$ reagent (Invitrogen; Thermo Fisher Scientific, Inc.) according to the manufacturer's protocol. Complementary (c)DNA was synthesized from RNA using a High Capacity cDNA Reverse Transcription kit (Applied Biosystems; Thermo Fisher Scientific, Inc.) and amplified using Power SYBR Green Master Mix (Applied Biosystems; Thermo Fisher Scientific, Inc.) on an Applied Biosystems Prism 7900HT sequence detection system (Applied Biosystems; Thermo Fisher Scientific, Inc.) according to the manufacturer's protocol. The PCR amplification was performed as follows: A total of 40 cycles at $95^{\circ} \mathrm{C}$ for $10 \mathrm{sec}$ and $65^{\circ} \mathrm{C}$ for $20 \mathrm{sec}$. U6 and GAPDH were used as the internal controls to normalize the expression of miR-181a and associated proteins in different groups using the $2^{-\Delta \Delta \mathrm{Cq}}$ method (19). Primer sequences were as follows: miR-181a-5p forward, 5'-ACACTCCAGCTGGGA ACATTCAACGCTGTCGG-3' and reverse, 5'-TGGTGTCGT GGAGTCGA-3'; U6 forward, 5'-CTCGCTTGGGCAGCA CA-3' and reverse, 5'-AACGCTTCACGAATTTGCGT-3'. KLF6 forward, 5'-CGGTGTGCTTTCGGAAGTG-3' and reverse, 5'-CGGTGTGCTTTCGGAAGTG-3'; Bcl-2 forward, 5'-TGGGATGCC TTTGTGGAACTAT-3' and reverse, 5'-AGAGACAGCCAGGAGAAATCA-3'; Bax forward, 5'-TCGTAGATCTATGGACGGGTCCGGGGAGCAGCT-3' and reverse, 5'-ATTAGCGGCCGCTCAGCCCATCTTCTT CCAGAT-3'; caspase-3 forward, 5'-TGTCATCTCGCTCTG GTACG-3' and reverse, 5'-AAATGACCCCTTCATCACCA-3'; GAPDH forward, 5'-AACTTTGGCATTGTGGAAGG-3' and reverse, 5'-GGAGACAACCTGGTCCTCAG-3'.

Western blot analysis. The treated cells were lysed in radioimmunoprecipitation assay buffer (Beyotime Institute of Biotechnology) containing protein inhibitor cocktail. The concentration of proteins was measured using a BCA Protein Assay kit (Thermo Fisher Scientific, Inc.). Proteins (10 $\mu \mathrm{g} / \mathrm{lane})$ were then separated via $10 \%$ SDS-PAGE (cat. no. S1052; Solarbio ${ }^{\circledR}$ Life Sciences) and transferred to a polyvinylidene difluoride membrane (Bio-Rad Laboratories, Inc.). After blocking in $5 \%$ non-fat milk with $0.1 \%$ Tris-buffered saline containing Tween 20 (10X TBST; cat. no. T1081; Solarbio ${ }^{\circledR}$ Life Sciences) for $1 \mathrm{~h}$ at room temperature, the membrane was then incubated with primary antibodies overnight at $4^{\circ} \mathrm{C}$. The following primary antibodies were used: Rabbit anti-KLF6 (1:1,000 dilution; cat. no. DF13114; Affinity Biosciences), rabbit anti-Bcl-2 (1:1,000 dilution; cat. no. ab32124), rabbit anti-Wnt1 (1:1,000 dilution; cat. no. ab15251), rabbit anti- $\beta$-catenin 
A

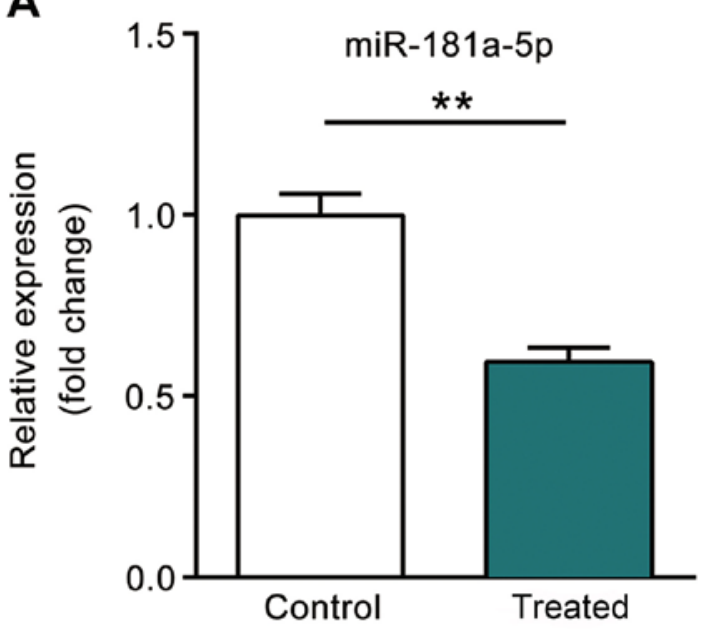

B

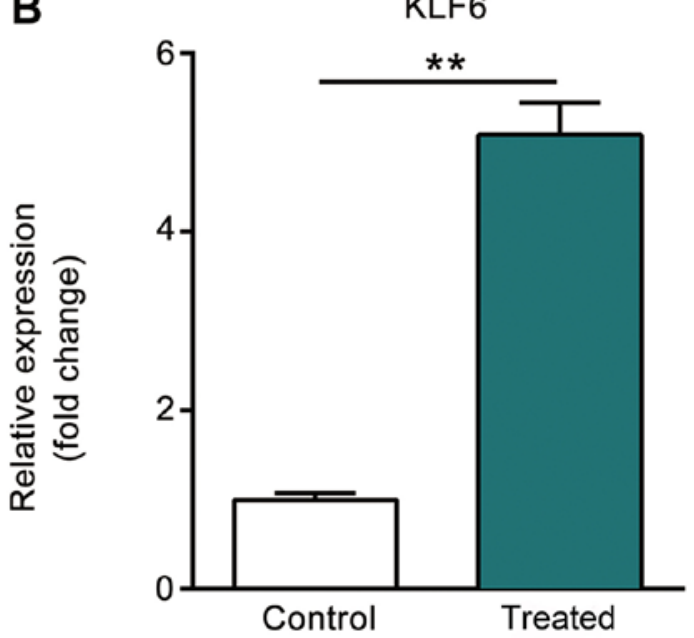

Figure 1. miR-181a-5p is significantly downregulated following treatment with high glucose. Expression levels of (A) miR-181a-5p and (B) KLF6 following treatment with high glucose. ${ }^{* *} \mathrm{P}<0.01$, treated vs. control group. miR, microRNA; KLF6, kruppel-like factor 6.

(1:5,000 dilution; cat. no. ab32572), rabbit anti-Bax $(1: 1,000$ dilution; cat. no. ab32503), rabbit anti-caspase-3 (1:500 dilution; cat. no. ab13847) and rabbit anti-GAPDH (1:10,000 dilution; cat. no. AB181602) (all from Abcam unless stated otherwise). Subsequently, the secondary antibodies IgG H\&L (Cy2 ${ }^{\circledR} ; 1: 1,000$ dilution; cat. no. ab6940; Abcam) were added, followed by incubation for $1 \mathrm{~h}$ at room temperature. Finally, NovexTM ECL Chemiluminescent Substrate Reagent Kit (WP2005; Thermo Fisher Scientific, Inc.) were used to detect bound antibodies by exposure to X-ray film. All results were normalized to the absorbance of the internal control GAPDH.

Luciferase activity assay. Following confirmation that KLF6 may be a predictive target gene of miR-181a using TargetScan, a luciferase reporter gene assay was performed. In brief, the target sequence purchased from GenePharma (Shanghai GenePharma Co., Ltd.) was inserted into the luciferase reporter plasmid pGL-3-Basic (Promega Corp.) recombinant reporter vector containing the predictive miR-181a-5p binding sequence. The mutant recombinant reporter vector was designed and synthesized by GenePharma (Shanghai GenePharma Co., Ltd.). Cells were then cultured in 24-well plates at a density of $2 \times 10^{4}$ cells/well until they reached $80 \%$ confluence. Subsequently, the cells were co-transfected with $0.2 \mu \mathrm{g}$ mutant or wild-type (WT) reporter plasmids and $10 \mathrm{nmol}$ miR-181a-5p mimics or miR-NC mimics using Lipofectamine ${ }^{\circledR} 2000$ (Invitrogen; Thermo Fisher Scientific, Inc.). At $48 \mathrm{~h}$ post-transfection, the harvested cells were analyzed using the Dual-luciferase Reporter Assay system (Promega Corp.) according to the manufacturer's protocol.

Statistical analysis. All of the aforementioned procedures were performed in triplicate. Values are expressed as the mean \pm standard deviation and differences were assessed using one-way analysis of variance, followed by the Newman-Keuls multiple-comparisons test. SPSS software (version 17.0; SPSS Inc.) was used for the statistical analyses and $\mathrm{P}<0.05$ was considered to indicate a statistically significant difference.

\section{Results}

miR-181a-5p expression is downregulated following treatment with high glucose. As presented in Fig. 1A, the expression level of miR-181a-5p was significantly downregulated following treatment with high glucose as compared with that in the control group. However, the expression level of KLF6, the predictive target gene of miR-181a-5p, was significantly upregulated following treatment with high glucose $(\mathrm{P}<0.01$; Fig. 1B).

KLF6 is a target of miR-181a-5p. As indicated in Fig. 2A, the 3'UTR of KLF6 mRNA contains a potential binding sequence for miR-181a-5p, which was identified using a bioinformatics analysis. A luciferase assay was subsequently performed using the GMCs in order to verify whether KLF6 is a target of miR-181a-5p. As presented in Fig. 2B, the relative luciferase activity was markedly decreased in the miR-181a-5p-transfected GMCs following co-transfection with the WT recombinant plasmid $(\mathrm{P}<0.01)$. However, there was no significant difference in luciferase activity between the miR-181a-5p and NC groups following co-transfection with the mutant recombinant plasmid.

Overexpression efficiency of miR-181a-5p and KLF6. The expression levels of miR-181a-5p and KLF6 were detected using RT-qPCR following transfection. As presented in Fig. 3A, the expression level of miR-181a-5p was significantly increased in the miR-181a-5p mimics group as compared with that in the $\mathrm{NC}$ and control groups $(\mathrm{P}<0.01)$. Furthermore, the expression level of KLF6 was markedly increased in the KLF6 group as compared with that in the pcDNA3.1 and control groups $(\mathrm{P}<0.01$; Fig. 3B).

miR-181a-5p suppresses GMC proliferation stimulated by high glucose. As presented in Fig. 4, after $48 \mathrm{~h}$ incubation, the proliferation of GMC cells was significantly decreased in the mimics group as compared with that in the $\mathrm{NC}$ or control 
A

\begin{tabular}{|c|c|}
\hline $\begin{array}{l}\text { Position } 403-409 \text { of KLF6 3' UTR } \\
\text { hsa-miR-181a-5p }\end{array}$ & $\begin{array}{l}5^{\prime} . . . \text { GGAAAAAAAGUAGUUUGAAUGUU... } \\
\text { 3' } \\
\text { 3, UGAGUGGCUGUCGCAACUUACAA }\end{array}$ \\
\hline f KLF6 3' & GGAAAAAAAGUAGUUCACUGACL \\
\hline
\end{tabular}

B

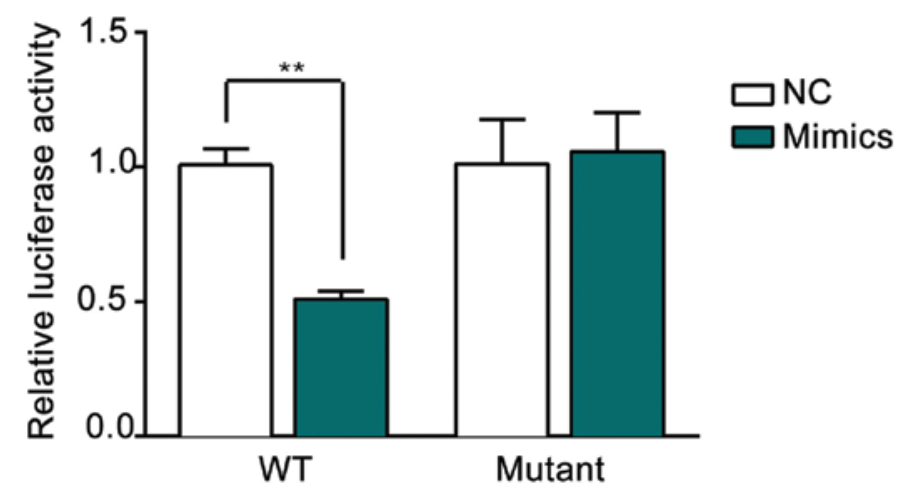

Figure 2. KLF6 is a target of miR-181a-5p. (A) The sequences of the WT of KLF6 3'UTR and the mutant position are presented. (B) Relative luciferase activity in different groups. ${ }^{* *} \mathrm{P}<0.01$, mimics vs. NC group. KLF6, kruppel-like factor 6; miR, microRNA; 3'UTR, 3'-untranslated region; hsa, Homo sapiens; NC, negative control (cells were treated with miR mimics control); WT, wild-type.

A

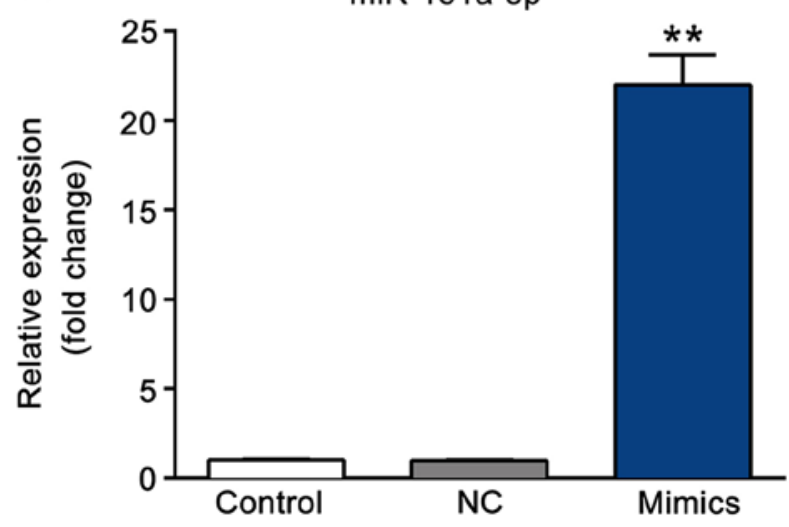

B

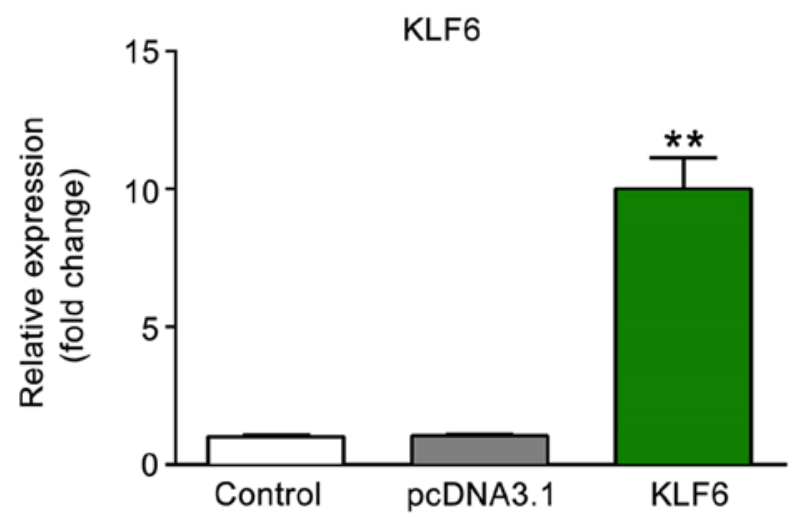

Figure 3. Transfection efficiency of miR-181a-5p and KLF6. Expression levels of (A) miR-181a-5p and (B) KLF6 in the different groups. ${ }^{* *} \mathrm{P}<0.01$, mimics vs. NC group or KLF6 vs. pcDNA3.1 group. miR, microRNA; KLF6, kruppel-like factor 6; NC, negative control (cells were treated with miR mimics control).

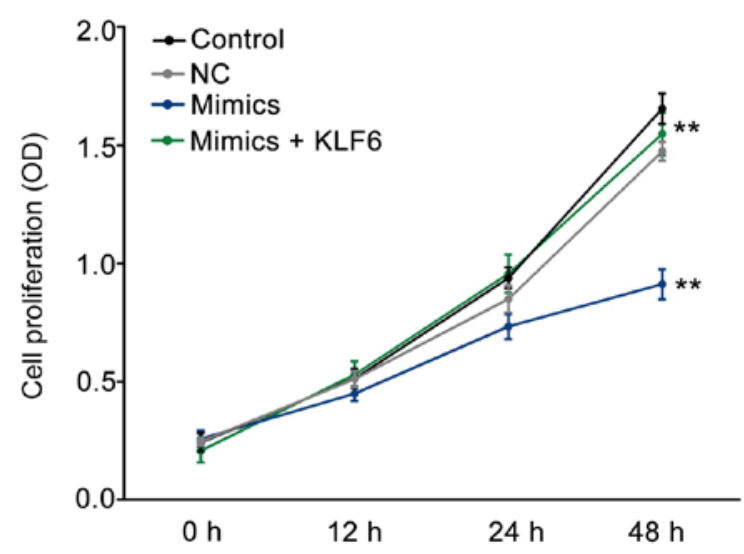

Figure 4. miR-181a-5p suppresses human glomerular mesangial cell proliferation. The cell viability in the different groups is presented. ${ }^{* *} \mathrm{P}<0.01$, mimics + KLF6 vs. mimics group or mimics vs. NC group. miR, microRNA; NC, cells were treated with miR mimics control negative control; OD, optical density. groups $(\mathrm{P}<0.01)$; this variation was reversed by co-transfection with pcDNA3.1-KLF6 $(\mathrm{P}<0.01)$.

Overexpression of KLF6 promotes human GMC proliferation. As presented in Fig. S1, the cell viability was prominently increased in the KLF6 group in comparison to that in the pcDNA3.1 and control groups $(\mathrm{P}<0.01)$.

miR-181a-5p promotes GMC apoptosis stimulated by high glucose. As presented in Fig. 5, cell apoptosis was significantly increased in the mimics group as compared with that in the $\mathrm{NC}$ or control groups $(\mathrm{P}<0.01)$; however, the variation was reversed by co-transfected with pcDNA3.1-KLF6 $(\mathrm{P}<0.01)$.

Overexpression of KLF6 suppresses human GMC apoptosis. As presented in Fig. S2, cell apoptosis was prominently 

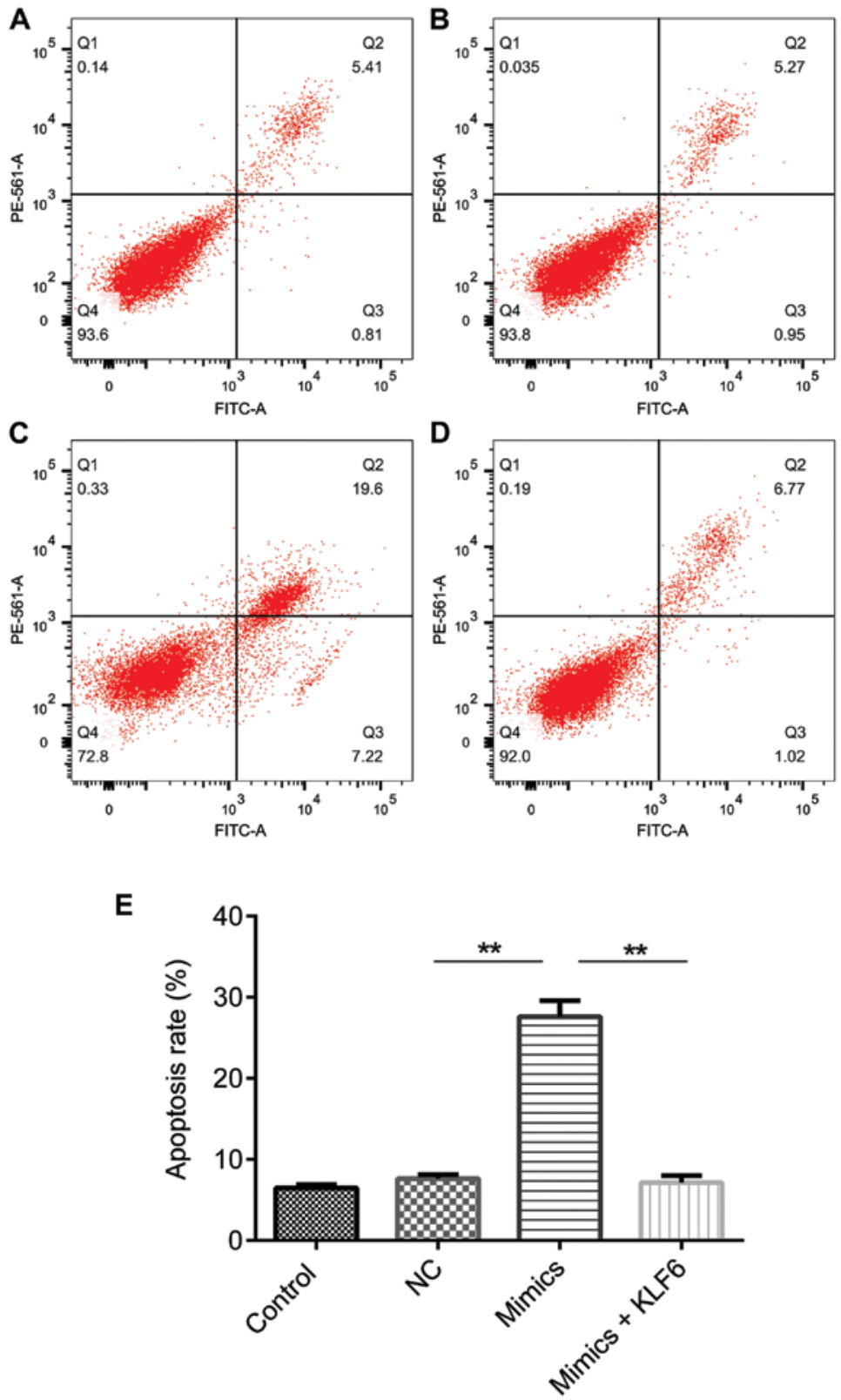

Figure 5. miR-181a-5p promotes human glomerular mesangial cell apoptosis. (A-D) representative flow cytometry dot plots for (A) control group, (B) NC group, (C) mimics group and (D) mimics + KLF6 group. (E) The corresponding results of A-D are presented. ${ }^{* *} \mathrm{P}<0.01$, mimics + KLF6 vs. mimics group or mimics vs. NC group. miR, microRNA; NC, negative control (cells were treated with miR mimics control); KLF6, kruppel-like factor 6; NC, negative control; Q, quadrant.

decreased in the KLF6 group as compared with that in the pcDNA3.1 and control groups $(\mathrm{P}<0.01)$.

mRNA expression levels of KLF6, Bcl-2, Bax and caspase-3 following transfection. As presented in Fig. 6A-D, the expression levels of KLF6 and Bcl-2 were markedly decreased, while Bax and caspase- 3 levels were increased in the mimics group as compared with those in the $\mathrm{NC}$ and control groups $(\mathrm{P}<0.01)$. However, the variation induced by transfection with miR-181a-5p mimics was reversed by co-transfection with pcDNA3.1-KLF6 $(\mathrm{P}<0.01)$.

miR-181a-5p may regulate GMC proliferation and apoptosis via the Wnt/ $\beta$-catenin signaling pathway. As presented in Fig. 7A and 7B, the protein expression levels of KLF6, Bcl-2, Wnt, $\beta$-catenin were significantly decreased, while Bax and caspase-3 were increased in the mimics group as compared with those in the $\mathrm{NC}$ and control groups. However, the variation was reversed by co-transfection with pcDNA3.1-KLF6.

KLF6 may regulate human GMC proliferation and apoptosis via the Wnt/ $\beta$-catenin signaling pathway. As presented in Fig. S3, the protein expression levels of KLF6, Bcl-2, Wnt and $\beta$-catenin were prominently increased, while Bax and caspase-3 were decreased in the KLF6 group as compared with those in the pcDNA3.1 and control groups.

\section{Discussion}

miR-181a-5p belongs to the miR-181a family, which has been reported to participate in malignant transformation (20-22) and various diseases (23-25). High glucose concentrations have 

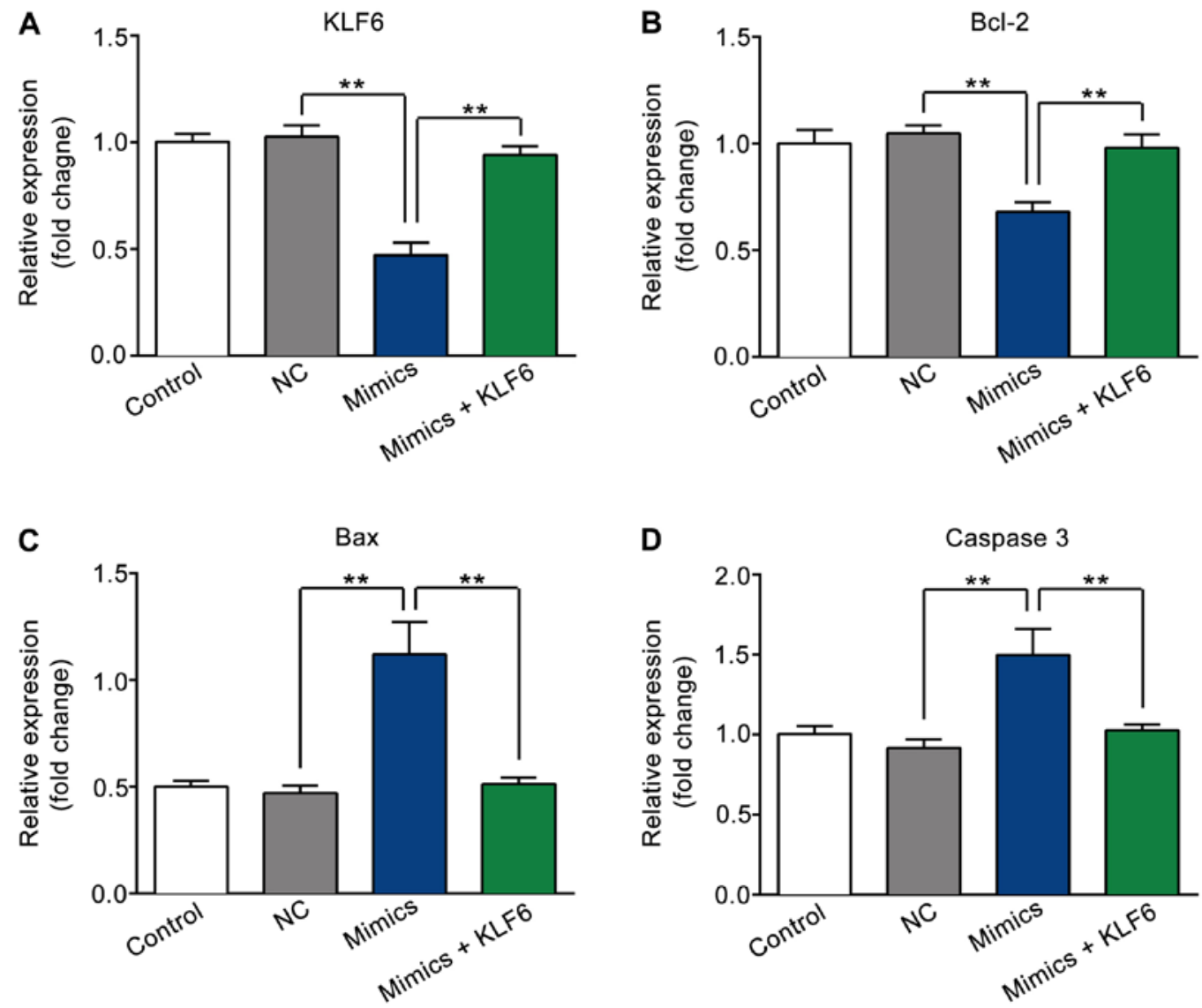

Figure 6. mRNA expression levels of KLF6, Bcl-2, Bax and caspase-3 following transfection. mRNA expression levels of (A) KLF6, (B) Bcl-2, (C) Bax and (D) caspase-3. ${ }^{* *} \mathrm{P}<0.01$, mimics + KLF6 vs. mimics group and mimic vs. NC group. KLF6, kruppel-like factor 6; NC, negative control (cells were treated with miR mimics control).

A

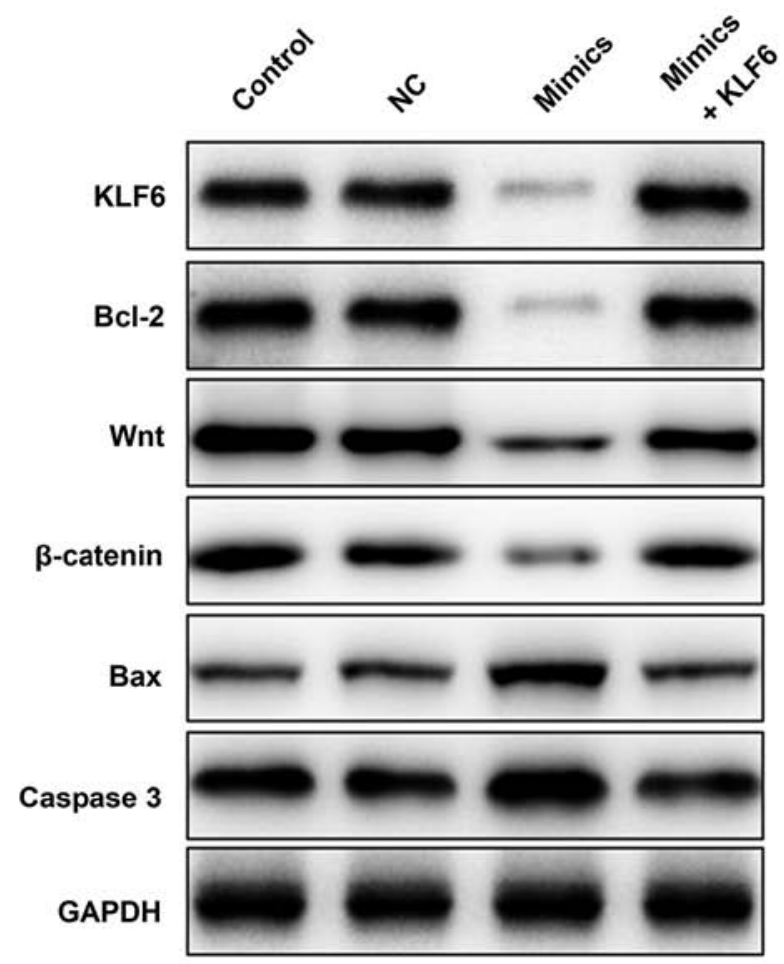

B

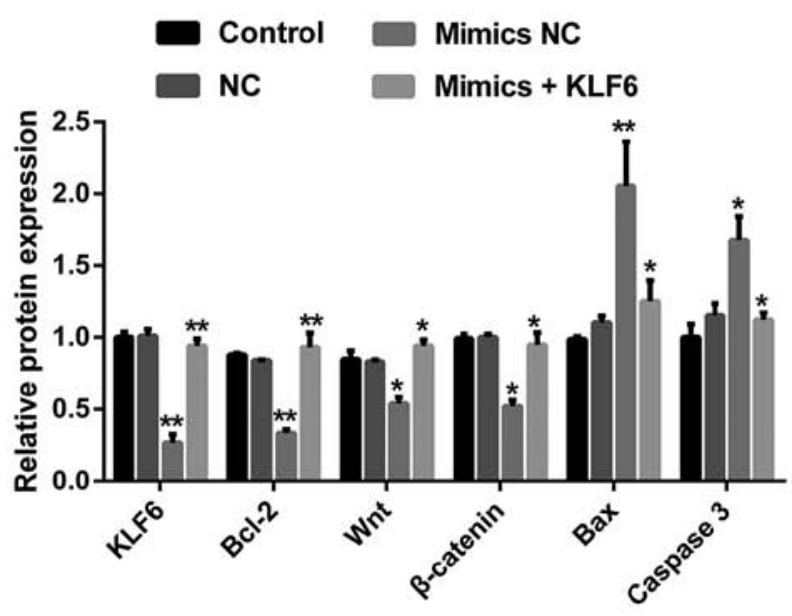

Figure 7. miR-181a-5p may regulate human glomerular mesangial cell proliferation and apoptosis via the Wnt/ $\beta$-catenin signaling pathway. (A) The protein expression levels of the associated factors in different groups were assessed by western blot analysis and (B) the results quantified. ${ }^{* *} \mathrm{P}<0.01$, " $\mathrm{P}<0.05$. miR, microRNA; KLF6, kruppel-like factor 6; cells were treated with miR mimics control NC, negative control (cells were treated with miR mimics control). 
been demonstrated to contribute to the uncontrolled proliferation of mesangial cells $(26,27)$ and high glucose was reported to be the principal cause of renal damage in diabetes (28), indicating that uncontrolled proliferation stimulated by high glucose in mesangial cells may contribute to the progression of DN. The results of the present study demonstrated that miR-181a-5p was highly expressed, while KLF6 exhibited low expression in GMCs following treatment with high glucose. Furthermore, overexpression of miR-181a-5p was indicated to inhibit the proliferation but promote apoptosis of GMCs stimulated by high glucose. Collectively, these results indicated that miR-181a-5p may have a pivotal in the genesis and progression of DN and may serve as a diagnostic marker.

Bcl-2 is a member of the Bcl-2 family, which is able to regulate cell apoptosis and functions as an anti-apoptotic protein $(29,30)$. Bax is a pro-apoptotic protein, which is activated in response to apoptotic stimuli (31). Caspase-3 is a member of the family of cysteine proteases, originally identified by their role in apoptosis, functioning as a pro-apoptotic protein in various cellular processes (32). KLF6 has been reported to be a regulator for the progression of $\mathrm{DN}$, functioning as a potential pathogenic factor in DN (33). The Wnt/ $\beta$-catenin signaling pathway, controlled by miRNAs, exerts its function to maintain proper cell-cell junctions and tissue homeostasis (34). Inactivation or inhibition of the Wnt/ $\beta$-catenin signaling pathway has been demonstrated to promote apoptosis and suppress cell proliferation in various cancer types $(35,36)$. Of note, a previous study reported that blocking the Wnt/ $\beta$-catenin signaling pathway may attenuate the proliferation of GMCs induced by high glucose levels (37). Consistent with previous studies, the results of the present study suggested that miR-181a-5p may be able to inhibit GMC proliferation but increase apoptosis by downregulating KLF6 and Bcl-2 expression and upregulating Bax and caspase-3 expression via the $\mathrm{Wnt} / \beta$-catenin signaling pathway. To the best of our knowledge, the present study was the first to demonstrate that miR-181a-5p could inhibit GMC proliferation while it promoted apoptosis by inhibition of KLF6 through Wnt/ $\beta$-catenin signaling pathway. However, there are some limitations in our study. In the future, we will conduct further experiments concerning the effect of high glucose on GMC proliferation and apoptosis. Meanwhile, more targets and underlying molecular mechanism remain to be further elucidated.

$\mathrm{Xu}$ et al (38) reported that miR-181a-5p expression was downregulated in rats with $\mathrm{DN}$ and may prevent fibrosis in HK-2 cells by targeting early growth response 1 . In the present study, miR-181a-5p overexpression was indicated to inhibit GMC proliferation but increase apoptosis by targeting KLF6 under high-glucose conditions. It was confirmed that KLF6 was a target gene of miR-181a-5p for the first time.

In conclusion, the present study indicated that miR-181a-5p was downregulated in GMCs following treatment with high glucose. Overexpression of miR-181a-5p inhibited GMC proliferation but promoted apoptosis at least partially through targeting KLF6 via the Wnt/ $\beta$-catenin signaling pathway. Overall, the results of the present study suggest that miR-181a-5p may have a crucial role in the occurrence and development of DN and may be a valuable diagnostic marker and therapeutic target for DN.

\section{Acknowledgements}

Not applicable.

\section{Funding}

No funding was received.

\section{Availability of data and materials}

The datasets used and/or analyzed during the current study are available from the corresponding author on reasonable request.

\section{Authors' contributions}

XL prepared the manuscript, performed some experiments and revised the manuscript for important content. WX performed the statistical analysis and helped with the study design, critical discussion and manuscript revision. All authors have read and approved the final manuscript.

\section{Ethics approval and consent to participate}

Not applicable.

\section{Patient consent for publication}

Not applicable.

\section{Competing interests}

The authors declare that they have no competing interests.

\section{References}

1. He L and Hannon GJ: MicroRNAs: Small RNAs with a big role in gene regulation. Nav Rev Genet 5: 522-531, 2004.

2. Bartel DP: MicroRNAs: Genomics, biogenesis, mechanism and function. Cell 116: 281-297, 2004.

3. Liu X, Li M, Peng Y, Hu X, Xu J, Zhu S, Yu Z and Han S: miR-30c regulates proliferation, apoptosis and differentiation via the Shh signaling pathway in P19 cells. Exp Mol Med 48: e248, 2016.

4. Vidal AF, Cruz AM, Magalhães L, Pereira AL, Anaissi AK, Alves NC, Albuquerque PJ, Burbano RM, Demachki S and Ribeiro-dos-Santos Â: hsa-miR-29c and hsa-miR-135b differential expression as potential biomarker of gastric carcinogenesis. World J Gastroenterol 22: 2060-2070, 2016.

5. Bansal N, Dhaliwal R and Weinstock RS: Management of diabetics in the elderly. Med Clin North Am 99: 351-377, 2015.

6. Nam S, Chesla C, Stotts NA, Kroon L and Janson SL: Barriers to diabetes management: Patient and provider factors. Diabetes Res Clin Pract 93: 1-9, 2011

7. Abboud HE: Mesangial cell biology. Exp Cell Res 318: 979-985, 2012.

8. Danesh FR, Sadeghi MM, Amro N, Philips C, Zeng L, Lin S, Sahai A and Kanwar YS: 3-Hydroxy-3-methylglutaryl CoA reductase inhibitors prevent high glucose-induced proliferation of mesangial cells via modulation of Rho GTPase/p21 signaling pathway: Implications for diabetic nephropathy. Proc Natl Acad Sci USA 99: 8301-8305, 2002.

9. Liu ZM, Zheng HY, Chen LH, Li YL, Wang Q, Liao CF and Li XW: Low expression of miR-203 promoted diabetic nephropathy via increasing TLR4. Eur Rev Med Pharmacol Sci 22: 5627-5634, 2018

10. Zhao D, Jia J and Shao H: iR-30e targets GLIPR-2 to modulate diabetic nephropathy: In vitro and in vivo experiments. J Mol Endocrinol 59: 181-190, 2017. 
11. Wang J, Duan L, Gao Y, Zhou S, Liu Y, Wei S, An S, Liu J, Tian L and Wang S: Angiotensin II receptor blocker valsartan ameliorates cardiac fibrosis partly by inhibiting miR-21 expression in diabetic nephropathy mice. Mol Med Endorcrinol 472: 149-158, 2018.

12. He YQ, Ding YL, Liang BY, Lin JJ, Kim TY, Yu HB, Hang HW and Wang $\mathrm{K}$ : A systematic study of dysregulated microRNA in type 2 diabetes mellitus. Int J Mol Sci 18: pii: 456, 2017.

13. Assmann TS, Recamonde-Mendoza M, De Souza BM and Crispim D: MicroRNA expression profiles and type 1 diabetes mellitus: Systematic review and bioinformatic analysis. Endocr Connect 6: 773-790, 2017.

14. Tetreault MP, Yang Y and Katz JP: Krüppel-like factors in cancer. Nat Rev Cancer 13: 701-713, 2013.

15. Huang X, Li X and Guo B: KLF6 induces apoptosis in prostate cancer cells through up-regulation of ATF3. J Biol Chem 283. 29795-29801, 2008.

16. Wahab AH, Kassem AM, Matter S, EI Deen AF, Helmy AS, Ismaeil MM and Zakaria MS: Role of KLF6 tumor suppressor gene mutations in the development of colorectal carcinoma in an Egyptian population. Hepatogastroenterology 57: 1405-1410, 2010.

17. Rane MJ, Zhao Y and Cai L: Krüppel-like factors (KLFs) in renal physiology and disease. EBioMedicine 40: 743-750, 2019.

18. Xu S, Lv YY, Zhao J, Wang JP, Zhao X and Wang S: Inhibitory effects of Shenkang injection and its main component emodin on the proliferation of high glucose-induced renal mesangial cells through cell cycle regulation and induction of apoptosis. Mol Med Rep 14: 3381-3388, 2016.

19. Livak KJ and Schmittgen TD: Analysis of relative gene expression data using real-time quantitative PCR and the 2(-Delta Delta C(T)) method. Methods 25: 402-408, 2001

20. Li Y, Kuscu C, Banach A, Zhang Q, Pulkoski-Gross A, Kim D, Liu J, Roth E, Li E, Shroyer KR, et al: MiR-181a-5p inhibits cancer cell migration and angiogenesis via downregulation of matrix metalloproteinase-14. Cancer Res 75: 2674-2685, 2015.

21. Taylor MA, Sossey-Alaoui K, Thompson CL, Danielpour D and Schiemann WP: TGF- $\beta$ upregulates miR-181a expression to promote breast cancer metastasis. J Clin Invest 123: 150-163, 2013.

22. Wei Y, Tao X, Xu H, Chen Y, Zhu L, Tang G, Li M, Jiang A, Shuai S, Ma J, et al: Role of miR-181a-5p and endoplasmic reticulum stress in the regulation of myogenic differentiation. Gene 592: 60-70, 2016.

23. Li S, Yang J, Xia Y, Fan Q and Yang KP: Long noncoding RNA NEAT1 promotes proliferation and invasion via targeting miR-181a-5p in non-small cell lung cancer. Oncol Res 26 : 289-296, 2018

24. Yu C, Sun J, Leng X and Yang J: Long noncoding RNA SNHG6 functions as a competing endogenous RNA by sponging miR-181a-5p to regulate E2F5 expression in colorectal cancer. Cancer Manag Res 11: 611-624, 2019.

25. Mi Y, Zhang D, Jiang W, Weng J, Zhou C, Huang K, Tang $H$, Yu Y, Liu X, Cui W, et al: miR-181a-5p promotes the progression of gastric cancer via RASSF6-mediated MAPK signaling activation. Cancer Lett 389: 11-22, 2017.
26. Zhang L, Pang S, Deng B, Qian L, Chen J, Zou J, Zheng J, Yang L, Zhang $C$, Chen $X$, et al: High glucose induces renal mesangial cell proliferation and fibronectin expression through $\mathrm{JNK} / \mathrm{NF}-\kappa \mathrm{B} / \mathrm{NADPH}$ oxidase/ROS pathway, which is inhibited by resveratrol. Int J Biochem Cell Biol 44: 629-638, 2012.

27. Sodhi CP, Phadke SA, Batlle D and Sahai A: Hypoxia and high glucose cause exaggerated mesangial cell growth and collagen synthesis: Role of osteopontin. Am J Physiol Renal Physiol 280: F667-F674, 2001.

28. Suzaki Y, Yoshizumi M, Kagami S, Nishiyama A, Ozawa Y, Kyaw M, Izawa Y, Kanematsu Y, Tsuchiya K and Tamaki T: BMK1 is activated in glomeruli of diabetic rats and in mesangial cells by high glucose conditions. Kidney Int 65: 1749-1760, 2004.

29. Singh L, Pushker N, Saini N, Sen S, Sharma A, Bakhshi S, Chawla B and Kashyap S: Expression of pro-apoptotic Bax and anti-apoptotic Bcl-2 proteins in human retinoblastoma. Clin Exp Ophthalmol 43: 259-267, 2015.

30. Zhu Y, Tchkonia T, Fuhrmann-Stroissnigg H, Dai HM, Ling YY, Stout MB, Pirtskhalava T, Giorgadze N, Johnson KO, Giles CB, et al: Identification of a novel senolytic agent, navitoclax, targeting the Bcl-2 family of anti-apoptotic factors. Aging Cell 15: 428-435, 2016.

31. Ghibelli L and Diederich M: Multistep and multitask Bax activation. Mitochondrion 10: 604-613, 2010.

32. Caruso Bavisotto C, Nikolic D, Marino Gammazza A, Barone R, Lo Cascio F, Mocciaro E, Zummo G, Conway de Macario E, Macario AJ, Cappello F, et al: The dissociation of the Hsp60/ pro-Caspase-3 complex by bis (pyridyl) oxadiazole copper complex (CubipyOXA) leads to cell death in NCI-H292 cancer cells. J Inorg Biochem 170: 8-16, 2017.

33. Qi W, Holian J, Tan CY, Kelly DJ, Chen XM and Pollock CA: The roles of Kruppel-like factor 6 and peroxisome proliferator-activated receptor- $\gamma$ in the regulation of macrophage inflammatory protein-3 $\alpha$ at early onset of diabetics. Int J Biochem Cell Biol 43: 383-392, 2011.

34. Ghahhari NM and Babashah S: Interplay between microRNAs and $\mathrm{Wnt} / \beta$-catenin signaling pathway regulates epithelial-mesenchymal transition in cancer. Eur J Cancer 51: 1638-1649, 2015.

35. Mao J, Fan S, Ma W, Fan P, Wang B, Zhang J, Wang H, Tang B, Zhang Q, Yu X, et al: Roles of Wnt/ $\beta$-catenin signaling in the gastric cancer stem cells proliferation and salinomycin treatment. Cell Death Des 5: e1039, 2014.

36. Gao L, Chen B, Li J, Yang F, Cen X, Liao Z and Long X: Wnt/ $\beta$-catenin signaling pathway inhibits the proliferation and apoptosis of U87 glioma cells via different mechanisms. PLoS One 12: e0181346, 2017.

37. Lan XQ, Wen HX, Aslam R, Marashi Shoshtari SS, Mishara A, Kumar V, Wang HC, Wu GS, Luo HR, Malhotra A and Singhal PC: Nicotine enhances mesangial cell proliferation and fibronectin production in high glucose milieu via activation of Wnt/ $\beta$-catenin pathway. Bioscience Rep 38: pii: BSR20180100, 2018.

38. Xu P, Guan MP, Bi JG, Wang D, Zheng ZJ and Xue YM: High glucose down-regulates microRNA-181a-5p to increase pro-fibrotic gene expression by targeting early growth response factor 1 in HK-2 cells. Cell Signal 31: 96-104, 2017. 